\title{
Lobbying with Two Audiences: Public vs Private Certification *
}

\author{
Frédéric KOESSLER ${ }^{\dagger}$
}

July 17,2006

\begin{abstract}
This note compares public and private information certification in a simple class of communication games with one sender and two receivers. It also emphasizes the role of belief consistency conditions in a perfect Bayesian equilibrium of such games.

Keywords: Certifiable information, cheap talk, consistency of beliefs.

JEL Classification: C72; D82.
\end{abstract}

\section{Introduction}

In this note we study a particular setting of lobbying activities with a single lobby and several decisionmakers. We refer to lobbying activities as meetings between the lobbyist (an informed interested party) and the decisionmakers in which the former try to influence the latter's choices by transmitting payoff-relevant information. In the simple setting introduced by Farrell and Gibbons [1989] — with two states of nature, two decisionmakers, and two actions each - we characterize the information revealed at equilibrium depending on whether meetings take place publicly or privately, and whether the information held by the lobbyist is certifiable or not.

Mutual discipline refers to a situation where information is revealed to neither decisionmaker when communication is private, but a fully revealing equilibrium exists when communication takes place publicly. The opposite situation, called mutual subversion, refers to a situation where there is a fully revealing equilibrium with each decisionmaker when communication takes place privately, but information is not revealed when communication is public. In Farrell and Gibbons's [1989] binary model, mutual discipline is possible in the cheap talk (non-certifiable) communication case, but there cannot be mutual subversion. We show that the opposite holds in the case of communication with certifiable information: there cannot be mutual discipline but mutual subversion is possible. From a theoretical point of view, our study emphasizes the role of belief consistency conditions that were irrelevant in previous work on strategic information revelation (e.g., Okuno-Fujiwara et al., 1990).

\footnotetext{
${ }^{*}$ I thank David Ettinger, Françoise Forges and Jérôme Mathis for interesting discussions. Financial support from an ACI grant by the French Ministry of Research is gratefully acknowledged.

†THEMA, Université de Cergy-Pontoise, 33 Boulevard du Port, F-95011 Cergy-Pontoise, France, E-mail: Frederic.Koessler@u-cergy.fr URL: www.u-cergy.fr/koessler/
} 


\section{Silent Game}

We consider one sender, $S$ (the lobbyist), and two receivers, $Q$ and $R$ (the decisionmakers). The sender observes the state $k \in\left\{k_{1}, k_{2}\right\}$, but the receivers do not. The prior probabilities of the states are $\operatorname{Pr}\left(k_{1}\right)=p \in(0,1)$ and $\operatorname{Pr}\left(k_{2}\right)=1-p$. As in Farrell and Gibbons [1989], $Q$ has two actions, $q_{1}$ and $q_{2}$, and $R$ has two actions, $r_{1}$ and $r_{2}$. The receivers' payoff, represented in the second coordinate of Table 1 , only depends on the state and their own action. The sender's payoff is the sum of the payoff he gets from $Q$ 's action and the payoff he gets from $R$ 's action. It is represented in the first coordinate of Table 1. For example, when $Q$ chooses action $q_{1}$ and $R$ chooses action $r_{2}$, the sender's payoff is $v_{1}+0$ if $k=k_{1}$, and $0+w_{2}$ if $k=k_{2}$. The parameters $x_{1}, x_{2}, y_{1}$ and $y_{2}$ are assumed strictly positive.

\begin{tabular}{c|c|c|c|c|}
\multicolumn{2}{c}{} & \multicolumn{2}{c}{$Q$} & \multicolumn{2}{c}{$R$} \\
\cline { 2 - 5 } \multicolumn{1}{c}{$k_{1}$} & $q_{1}$ & $q_{2}$ & $r_{1}$ & $r_{2}$ \\
\cline { 2 - 5 }$k_{2}, x_{1}$ & 0,0 & $w_{1}, y_{1}$ & 0,0 \\
\cline { 2 - 5 }$k_{2}$ & 0,0 & $v_{2}, x_{2}$ & 0,0 & $w_{2}, y_{2}$ \\
\cline { 2 - 5 } & & &
\end{tabular}

Table 1: Silent game between the lobbyist and the two decisionmakers.

Without communication, the optimal actions of the decisionmakers are

$$
q(p)=\left\{\begin{array}{ll}
q_{1} & \text { if } p \geq \bar{x} \equiv \frac{x_{2}}{x_{1}+x_{2}}, \\
q_{2} & \text { if } p \leq \bar{x},
\end{array} \quad \text { and } \quad r(p)= \begin{cases}r_{1} & \text { if } p \geq \bar{y} \equiv \frac{y_{2}}{y_{1}+y_{2}} \\
r_{2} & \text { if } p \leq \bar{y}\end{cases}\right.
$$

\section{Cheap Talk}

We consider the direct cheap talk extension of the silent game in which before actions are taken, but after the state is revealed to the lobbyist, the latter can, whatever his type, send a costless message in $M=\left\{m_{1}, m_{2}\right\}$ to the decisionmakers. We say that communication is $p u b$ lic when both decisionmakers observe the same message from the lobbyist. On the contrary, when the lobbyist can send a private, possibly different message to each decisionmaker, then communication is said private. ${ }^{1}$ A perfect Bayesian equilibrium is defined as usual. In the cheap talk games considered in this section, the set of perfect Bayesian equilibrium outcomes coincide with the set of Nash equilibrium outcomes. These sets may differ in the case of certifiable information (see the next section).

When communication is public, the receivers' beliefs about the state should be the same since the receivers start exactly with the same prior beliefs, and the lobbyist's message is common knowledge. This is indeed true along any Nash equilibrium path (by Bayes' rule given the lobbyist's strategy), but also off the equilibrium path at a perfect Bayesian equilibrium

\footnotetext{
${ }^{1}$ The fact that a decisionmaker knows whether the lobbyist sends a message to the other decisionmaker is irrelevant because there is no payoff interaction between the two decisionmakers. In more general settings, private communication may differ from secret communication.
} 
(see, e.g., Fudenberg and Tirole, 1991, condition B(iv) page 332). ${ }^{2}$ Since Nash and perfect Bayesian equilibrium outcomes coincide in cheap talk games, this last requirement is irrelevant here but will matter in the associated information certification games.

We assume without loss of generality that $\bar{x}<\bar{y}$. Then, the decisionmakers' optimal actions given a (common) belief $\mu$ about state $k_{1}$ are

$$
(q(\mu), r(\mu))= \begin{cases}\left(q_{1}, r_{1}\right) & \text { if } \mu \geq \bar{y} \\ \left(q_{1}, r_{2}\right) & \text { if } \bar{x} \leq \mu \leq \bar{y} \\ \left(q_{2}, r_{2}\right) & \text { if } \mu \leq \bar{x}\end{cases}
$$

A fully revealing equilibrium is a (Nash or perfect Bayesian) equilibrium in which the lobbyist reveals all his information, for example by sending message $m_{1}$ when the state is $k_{1}$ and $m_{2}$ when the state is $k_{2}$. We immediately get the following proposition (Farrell and Gibbons, 1989).

Proposition 1 Consider the cheap talk game. There exists a fully revealing equilibrium when the lobbyist communicates privately with the decisionmaker $Q$ ( $R$, respectively) if and only if $v_{1} \geq 0$ and $v_{2} \geq 0$ ( $w_{1} \geq 0$ and $w_{2} \geq 0$, respectively). There exists a fully revealing equilibrium when the lobbyist communicates publicly with the two decisionmakers if and only if $v_{1}+w_{1} \geq 0$ and $v_{2}+w_{2} \geq 0$.

Therefore, whenever there is a fully revealing equilibrium in each private cheap talk game, then there is also one in the public cheap talk game. In Farrell and Gibbons's [1989] terms, mutual subversion (full revelation with both receivers in private but not in public) is not possible in this setting. However, the unique equilibrium outcome may be non-revealing with both receivers in private, but a fully revealing equilibrium may exist in public (take, e.g., $v_{1}=w_{2}=3$ and $\left.v_{2}=w_{1}=-1\right) ;^{3}$ this situation is called mutual discipline. ${ }^{4}$

\section{Information Certification}

When the set of messages available to the lobbyist depends on his type, information is certifiable as in, e.g., Green and Laffont [1986], Okuno-Fujiwara et al. [1990], Seidmann and Winter [1997] or Forges and Koessler [2005]. To simplify the exposition, we assume that each state is certifiable, so the set of messages available to the lobbyist is $M\left(k_{1}\right)=\left\{m_{1}, \bar{m}\right\}$ when the state is $k_{1}$ and $M\left(k_{2}\right)=\left\{m_{2}, \bar{m}\right\}$ when the state is $k_{2}$. Of course, in this setting the conditions for a fully revealing equilibrium to exist are weaker than in the cheap talk case

\footnotetext{
${ }^{2}$ It is easy to see that a common belief for the receivers off the equilibrium path is a requirement of Kreps and Wilson's [1982] sequential equilibrium and Selten's [1975] perfect equilibrium because in the perturbed games receivers use the same trembling strategies of the sender to update their beliefs.

${ }^{3}$ Partially revealing equilibria, in mixed strategies, may also exist for generic parameters.

${ }^{4} \mathrm{~A}$ recent application of this effect includes, e.g., Levy and Razin [2004], in a binary model of conflict resolution between two countries in which (cheap talk) communication concerns the cost-benefit ratio from making concessions.
} 
since now a lobbyist's type is not necessarily able to imitate the other type's message. As the following proposition shows, the relationship between these conditions in the public and private communication situations is also different, and depends on the adopted equilibrium concept.

Proposition 2 Consider the information certification game. There exists a fully revealing Nash or perfect Bayesian equilibrium when the lobbyist communicates privately with the decisionmaker $Q$ ( $R$, respectively) if and only if $v_{1} \geq 0$ or $v_{2} \geq 0\left(w_{1} \geq 0\right.$ or $w_{2} \geq 0$, respectively). There exists a fully revealing Nash equilibrium when the lobbyist communicates publicly with the two decisionmakers if and only if condition (i), (ii), (iii) or (iv) below holds. There exists a fully revealing perfect Bayesian equilibrium when the lobbyist communicates publicly with the two decisionmakers if and only if condition (i), (ii) or (iii) below holds.

$$
\begin{array}{ll}
\text { (i) } v_{1}+w_{1} \geq 0 & \text { (ii) } v_{2}+w_{2} \geq 0 \\
\text { (iii) } w_{1} \geq 0 \text { and } v_{2} \geq 0 & \text { (iv) } v_{1} \geq 0 \text { and } w_{2} \geq 0
\end{array}
$$

Proof. The fully revealing Nash equilibrium in the private communication game with $Q$ is supported by the fully revealing strategy $\left(m_{1}\left|k_{1}, m_{2}\right| k_{2}\right)$ and the off the equilibrium path action $q_{1} \mid \bar{m}$ if $v_{2} \geq 0, q_{2} \mid \bar{m}$ if $v_{1} \geq 0$, and any of the two if $v_{1}, v_{2} \geq 0$. If $v_{1}, v_{2}<0$ then, whatever the mixed action (in $\Delta\left(\left\{q_{1}, q_{2}\right\}\right)$ of $Q$ after message $\bar{m}$, the sender has a strict incentive to deviate from full information revelation. To see that the fully revealing Nash equilibrium is also a perfect Bayesian equilibrium it suffices to remark that action $q_{1} \mid \bar{m}$ can be made sequentially rational with a belief $\mu\left(k_{1} \mid \bar{m}\right)$ that puts, e.g., probability one to $k_{1}$ after message $\bar{m}$, and action $q_{2} \mid \bar{m}$ with $\mu\left(k_{1} \mid \bar{m}\right)=0$. The same proof applies for the private communication game with $R$.

Next, consider the Nash equilibria of the public communication game. Full information revelation is supported by the following actions of the receivers in the different situations: (i) $\left(q_{2}, r_{2}\right)$, (ii) $\left(q_{1}, r_{1}\right)$, (iii) $\left(q_{1}, r_{2}\right)$ and (iv) $\left(q_{2}, r_{1}\right)$. We must also show that there is no fully revealing Nash equilibrium when neither of the conditions above are satisfied. In that case, four situations are possible: (a) $v_{2}+w_{2}<0$ and $v_{1}, w_{1}<0$, (b) $v_{1}+w_{1}<0, v_{2}+w_{2}<0$ and $w_{1}, w_{2}<0$, (c) $v_{1}+w_{1}<0, v_{2}+w_{2}<0$ and $v_{1}, v_{2}<0$, and (d) $v_{1}+w_{1}<0$ and $v_{2}, w_{2}<0$. In situation (a), since $v_{1}, w_{1}<0$, type $k_{1}$ does not deviate only if the receivers play $\left(q_{1}, r_{1}\right) \mid \bar{m}$. But then, since $v_{2}+w_{2}<0$, type $k_{2}$ deviates and sends message $\bar{m}$ instead of $m_{2}$. Situation (d) is symmetric. Next, consider situation (b). If $v_{1}<0$ or $v_{2}<0$ we are also in situation (a) or (d), so assume that $v_{1}, v_{2} \geq 0$. The sender does not deviate from full information revelation if and only if there is a mixed actions profile of the receivers, ${ }^{5}\left(\alpha_{1}, \alpha_{2}\right)$, $\left(\beta_{1}, \beta_{2}\right)$, where $\alpha_{i}$ is $Q$ 's probability of playing action $q_{i}$ after $\bar{m}$, and $\beta_{i}$ is $R$ 's probability of

\footnotetext{
${ }^{5}$ It can be checked that the result also holds when the receivers can use correlated strategies.
} 
playing action $r_{i}$ after $\bar{m}$, such that

$$
\begin{aligned}
& v_{1}+w_{1} \geq \alpha_{1} \beta_{1}\left(v_{1}+w_{1}\right)+\alpha_{1} \beta_{2} v_{1}+\alpha_{2} \beta_{1} w_{1}, \\
& v_{2}+w_{2} \geq \alpha_{1} \beta_{2} w_{2}+\alpha_{2} \beta_{1} v_{2}+\alpha_{2} \beta_{2}\left(v_{2}+w_{2}\right) .
\end{aligned}
$$

If $v_{1}=0$ or $v_{2}=0$, then these conditions cannot be satisfied simultaneously, so let $v_{1}, v_{2}>0$. The previous inequalities become equivalent to (recall that $v_{1}>0$ and $w_{2}<0$ )

$$
\begin{aligned}
& \alpha_{1} \beta_{2} \leq\left(1-\alpha_{1} \beta_{1}\right)+\left(w_{1} / v_{1}\right)\left(1-\alpha_{1} \beta_{1}-\alpha_{2} \beta_{1}\right), \\
& \alpha_{1} \beta_{2} \geq\left(1-\alpha_{2} \beta_{2}\right)+\left(v_{2} / w_{2}\right)\left(1-\alpha_{2} \beta_{2}-\alpha_{2} \beta_{1}\right) .
\end{aligned}
$$

Since $v_{2}+w_{2}<0, w_{2}<0$ and $v_{2}>0$ imply $v_{2} / w_{2}>-1$, and $v_{1}+w_{1}<0$ and $v_{1}>0$ imply $w_{1} / v_{1}<-1$, for a fully revealing Nash equilibrium to exist we must have $\left(1-\alpha_{2} \beta_{2}\right)+$ $(-1)\left(1-\alpha_{2} \beta_{2}-\alpha_{2} \beta_{1}\right) \leq\left(1-\alpha_{1} \beta_{1}\right)+(-1)\left(1-\alpha_{1} \beta_{1}-\alpha_{2} \beta_{1}\right)$. This inequality is strict if $\left(1-\alpha_{2} \beta_{2}-\alpha_{2} \beta_{1}\right)$ or $\left(1-\alpha_{1} \beta_{1}-\alpha_{2} \beta_{1}\right)$ equals zero. If the first (second, respectively) term is zero, then type $k_{2}\left(k_{1}\right.$, respectively) deviates. But strict inequality implies $\alpha_{2} \beta_{1}<\alpha_{2} \beta_{1}$, a contradiction. Therefore, there is no fully revealing Nash equilibrium. Situation (c) is symmetric.

Finally, consider the perfect Bayesian equilibria of the public communication game. The actions profiles $\left(q_{2}, r_{2}\right)\left|\bar{m},\left(q_{1}, r_{1}\right)\right| \bar{m}$ and $\left(q_{1}, r_{2}\right) \mid \bar{m}$, respectively, can be made sequentially rational with the common off the equilibrium path beliefs $\mu\left(k_{1} \mid \bar{m}\right) \leq \bar{x}, \mu\left(k_{1} \mid \bar{m}\right) \geq \bar{y}$ and $\mu\left(k_{1} \mid \bar{m}\right) \in[\bar{x}, \bar{y}]$, respectively. Hence, we get a fully revealing perfect Bayesian equilibrium in cases (i) to (iii). It remains to show that for any sequentially rational mixed actions profile of the receivers after message $\bar{m}$ (with a common belief), the sender deviates in case (iv) if neither (i), (ii) nor (iii) are satisfied. This situation implies $v_{1}+w_{1}<0, v_{2}+w_{2}<0$ and $v_{1}, v_{2} \geq 0$. Since a sequentially rational mixed actions profile with common beliefs puts zero probability on $\left(q_{2}, r_{1}\right)$ (see the best responses in Equation (1)), it is clear that in this situation both types of the sender want to deviate by sending message $\bar{m}$.

Compared to the cheap talk situation, mutual discipline is impossible (whatever the equilibrium concept). Indeed, there is no fully revealing equilibrium in the private meetings if and only if $v_{1}, v_{2}, w_{1}$ and $w_{2}$ are strictly negative, which implies that conditions (i) to (iv) are not satisfied. On the contrary, mutual subversion becomes possible with the perfect Bayesian equilibrium concept (but not with the Nash equilibrium concept). For example, when $v_{1}=w_{2}=x_{1}=x_{2}=y_{1}=1, v_{2}=w_{1}=-2$ and $y_{2}=2$ there is a fully revealing perfect Bayesian equilibrium in both private meetings but there is none in the public meeting.

Yet, relaxing the definition of perfect Bayesian equilibrium by allowing any belief off the equilibrium path changes this last conclusion. Indeed, the actions profile $\left(q_{2}, r_{1}\right) \mid \bar{m}$ can be made sequentially rational with heterogeneous beliefs $\mu_{Q}\left(k_{1} \mid \bar{m}\right) \leq \bar{x}$ for receiver $Q$ and $\mu_{R}\left(k_{1} \mid \bar{m}\right) \geq \bar{y}>\bar{x}$ for receiver $R$. Hence, under this weaker but less standard definition, perfect Bayesian equilibrium and Nash equilibrium outcomes coincide. 


\section{Conclusion}

In this note we have studied strategic information certification in a simple model of communication with heterogeneous audiences. In general, contrary to the cheap talk case, public communication with certifiable information interfere with information revelation comparing to the case of private communication, at least under the standard assumption of perfect Bayesian equilibrium behavior. How this conclusion holds and depends on belief consistency requirements in more general settings is left for further research.

\section{References}

J. Farrell and R. Gibbons. Cheap talk with two audiences. American Economic Review, 79: 1214-1223, 1989.

F. Forges and F. Koessler. Communication equilibria with partially verifiable types. Journal of Mathematical Economics, 41(7):793-811, 2005.

D. Fudenberg and J. Tirole. Game Theory. MIT Press, 1991.

J. R. Green and J.-J. Laffont. Partially verifiable information and mechanism design. Review of Economic Studies, 53(3):447-456, 1986.

D. M. Kreps and R. Wilson. Sequential equilibria. Econometrica, 50(4):863-894, 1982.

G. Levy and R. Razin. It takes two: An explanation for the democratic peace. Journal of the European Economic Association, 2(1):1-29, 2004.

A. Okuno-Fujiwara, M. Postlewaite, and K. Suzumura. Strategic information revelation. Review of Economic Studies, 57:25-47, 1990.

D. J. Seidmann and E. Winter. Strategic information transmission with verifiable messages. Econometrica, 65(1):163-169, 1997.

R. Selten. Reexamination of the perfectness concept for equilibrium points in extensive games. International Journal of Game Theory, 4(1):25-55, 1975. 